\title{
New Tetraquark Spotted in Electron-Positron Collisions
}

\author{
The detection of a new particle containing both charm and strange quarks \\ could offer new insights into how hadrons form.
}

\section{By Sophia Chen}

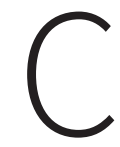
reating particles composed of novel combinations of quarks in high-energy particle collisions lets physicists develop theories of quantum chromodynamics, which describe how quarks and gluons interact. Now, the BESIII Collaboration at the Beijing Electron Positron Collider in China has detected another example of such a combination-a "tetraquark" called $Z_{c s}[\mathbf{1}]$. The result offers insight into how quarks are distributed inside a hadron.

The $Z_{c s}$ tetraquark is a charged particle consisting of four quarks: a charm quark, a charm antiquark, a strange quark, and an up antiquark. Theoretical models predicting the existence of this particle also suggest that its component quarks could produce more combinations of quantum states than those in other well-known particles. This is because, compared with three-quark baryons and two-quark mesons, for example, $Z_{c s}$ possesses more possible internal configurations: the quarks might be distributed evenly, as a diquark-antidiquark pair, or they could be in a loosely bound "molecular" state.

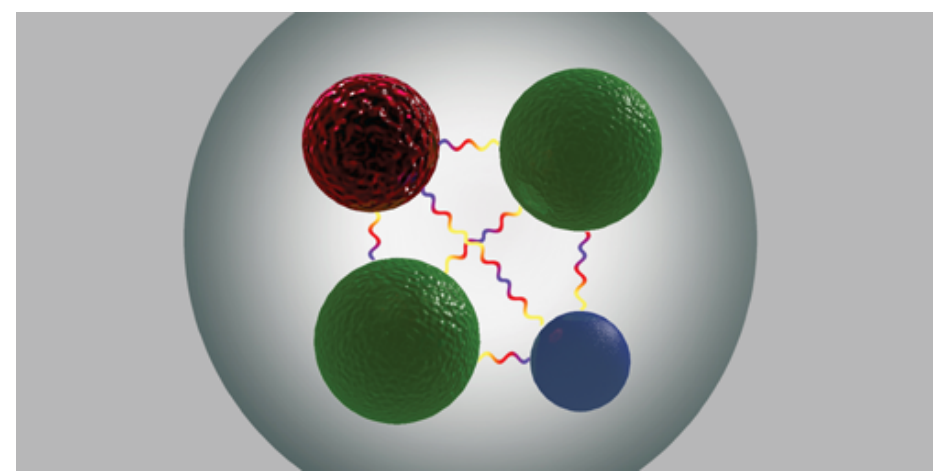

Credit: Xiao-Rui Lyu/University of Chinese Academy of Sciences
The researchers spotted the new tetraquark by measuring the momenta of primary particles and decay products generated in electron-positron collisions. Using Monte Carlo simulations to reconstruct the decay pathways from these measurements, they were able to pick out a signal corresponding to the predicted $Z_{c s}$ tetraquark's mass of about $3.98 \mathrm{GeV} / c^{2}$, or 4 times that of the proton. This signal was detected at the 5.3-sigma level, which is beyond the certainty threshold that constitutes a discovery.

To better understand the $Z_{c s}$ tetraquark, the team plans to collect more data in a range around the particle energy. These measurements will let them explore the particle's production and decay mechanisms and search for its excited states and its neutral counterpart.

Correction (23 March 2021): An earlier version stated an incorrect number for the energy targeted by planned experiments.

Sophia Chen is a freelance science writer based in Columbus, Ohio.

\section{REFERENCES}

1. M. Ablikim et al. (BESIII Collaboration), "Observation of a near-threshold structure in the $K^{+}$recoil-mass spectra in $e^{+} e^{-} \rightarrow K^{+}\left(D_{s}^{-} D^{* 0}+D_{s}^{*-} D^{0}\right)$," Phys. Rev. Lett. 126, 102001 (2021). 